Saudi Journal of Biomedical Research

Abbreviated Key Title: Saudi J Biomed Res ISSN 2518-3214 (Print) |ISSN 2518-3222(Online)

\title{
Albendazole Reduces Serum Levels of Inflammatory Cytokines: Potential Strategy for the Management of Cytokine Storm
}

AbdulFattahAdekunleOnifade, GaniyuOlatunbosunArinola*

Department of Immunology, University Of Ibadan, Nigeria

DOI:10.36348/sjbr.2020.v05i11.006 | Received: 22.10.2020 | Accepted: 01.11.2020| Published: 14.11 .2020

*Corresponding author: Ganiyu Olatunbosun Arinola

\section{Abstract}

Background: The phenomenon of cytokine storm is responsible for the pathogenesis and severity of COVID-19, which lacks specific drug treatment and vaccine for prevention. Thus, this report suggested that albendazole anthelminthic drug administration might reduce cytokine storm in COVID-19 patients. Method: This longitudinal study assessed serum levels of cytokines (IFN- $\gamma$, IL-4 and IL-8) usig ELISA in apparently healthy Nigerian adults before and 4 weeks after oral 400mg single dose of albendazole anthelminthic drug administration. Data presented as Median (Interquartile range) were analysed using Wilcoxon Signed Ranks Test, with levels of significance set at $\alpha_{0.05}$. Results: Serum median levels of IFN- $\gamma$ and IL-8 were significantly reduced at 4 weeks post- albendazole administration (p < 0.05 ) while IL-4 was insignificantly reduced at 4 weeks post- albendazole administration $(\mathrm{p}>0.05)$ compared with median levels before albendazole administration. Conclusion: Since albendazole administration reduces serum IFN- $\gamma$ and IL- 8 which are involved in COVID-19 cytokine storm, it is suggested that albendazole has potential for the management of cytokine storm in COVID-19 patients. This however needs further clarification.

Keywords: Albendazole, COVID-19, Cytokine storm.

Copyright (C) 2020 The Author(s): This is an open-access article distributed under the terms of the Creative Commons Attribution 4.0 International License (CC BY-NC 4.0) which permits unrestricted use, distribution, and reproduction in any medium for non-commercial use provided the original author and source are credited.

\section{INTRODUCTION}

Despite ongoing intensive research on prevention, control and management of COVID-19 pandemic, no known specific and effective drugs or vaccines have been developed to manage or prevent COVID-19 [1-3]. Therefore, many drugs have been evaluated in its management and the search continues. Hosseinet al., [1] reported that based on the preliminary data from clinical trials and considering the National Institute of Health (NIH) and Food and Drug Administration (FDA) recommendation, remdesivir and convalescent blood products are the most promising potential for COVID-19 treatment. The group [1] added that the uses of chloroquine, hydroxychloroquine, favipiravir, ivermectin, and colchicine might also be effective. Tocilizumab was deemed as adjunctive therapy for patients with cytokine release syndrome while baricitinib and ruxolitinib were reported to have additive immunosuppressive effect [1]. Santos et al., [2] opined that therapeutics for COVID-19 with particularly high potential efficacy for repurposing includes camostat mesylate, remdesivir, favipiravir, tocilizumab, baricitinib, convalescent plasma, and humanized monoclonal antibodies. Camostat mesylate showed therapeutic potential, likely by preventing viral entry into epithelial cells. Another group [3] showed that antivirals remdesivir and favipiravir appeared beneficial to COVID-19 patients by decreasing viral replication suggesting that remdesivir speeds recovery from COVID-19 while tocilizumab and baricitinib improved mortality by preventing a severe cytokine storm. Convalescent plasma and humanized monoclonal antibodies was shown to offer passive immunity and decreased recovery time. These previous literatures show that searching for specific drug to treat COVID-19 is continuous and that drugs used for the management of COVID-19 patients were not originally developed to treat novel SARS-COV 2 infection suggesting opportunities for further drug researches.

During SARS-COV infection of host cells, pathogen recognition receptors (PRRs) recognize the viral nucleic acid before translocated to the nucleus and promote the synthesis of type 1 interferons (IFNs). Type 1 IFNs subsequently activate downstream JAKSTAT signal pathway and promote the expression of IFNstimulated genes (ISGs)[4]. T-helper cells produce proinflammatory cytokines via the NFk-B signaling pathway. IL-17 cytokine recruit monocytes and neutrophils to the site of infection with 
inflammation and activate other downstream cytokine and chemokine cascades, such as IL-1, IL-6, IL-8, IL21 and TNF- $\beta$ [5]. Increased cytokine production characterized severe COVID-19, thus most severe COVID-19 cases exhibit an extreme increase in inflammatory cytokines, including IL-1 $\beta$, IL-2, IL-6, IL-7, IL-8, IL-10, granulocyte-colony stimulating factor (G-CSF), granulocyte macrophage-colony stimulating factor (GM-CSF), interferon-inducible protein-10 (IP10), monocyte chemotactic protein 1 (MCP1), macrophage inflammation protein- $1 \alpha$, IFN- $\gamma$, and TNF$\alpha$ [6-9] representing a "cytokine storm" $[10,11]$. Other study showed that cytokine levels, including IL2, IL-4, IL-6, IL-10, TNF- $\alpha$ and IFN- $\gamma$, are elevated in severe and critical COVID-19 cases [12]. In non-severe patients, cytokines such as IL-1 $\beta$, IL-1RA, IL-2R, IL-6, IL-7, IL-8, IL-9, IL-10, IFN- $\gamma$, TNF- $\alpha$, G-CSF, GMCSF, IP10 and MCP1 were up-regulated in COVID patients [9-11] but were significantly lower than levels in severe COVID patients. Studies have indicated that up-regulation of expression of pro-inflammatory molecules contribute to the pathogenesis of SARS [13], therefore reduction of cytokine storm during COVID19 is suggested as part of its management strategy.

Albendazole (a benzimidazolecarbamate) has been in clinical use for almost three decades as a safe, relatively cheap, pharmacologically-effective and oral anthelmintic drug with minimal side effects [14]. The primary mode of action of albendazole in susceptible parasites has been described as binding to $\beta$-tubulin and leading to inhibition of microtubule polymerization [15]. Albendazole was reported as a potent inhibitor of a wide variety of cancer cell lines, hepatocellular cancer cells, colorectal, pancreatic, ovarian, and a number of other human cancer cells $[16,17]$. Some patients with colorectal cancer and liver metastases had shown a decline or stabilization of tumor marker (carcinoembryonic antigen) when treated with oral albendazole [17]. Albendazole treatment decreased IL10 production, improved cellular immunity and ultimately improved in CD4+ T cell counts in HIV-1 patients [18]. Leung et al., [19] reported that anthelminthic treatments alter the incidence of diarrheal disease caused by viral and bacterial pathogens in school children in southern Vietnam.

In another study, Anuradha et al., [20] confirmed that the modulation of systemic and tuberculosis antigen-stimulated cytokine responses in $S$. stercoralis-latent tuberculosis co-infection is reversible (for the most part) by anthelmintic treatment. Apart from therapeutic agent, support managements such as micronutrients, antioxidants and exercise therapy were recommended for the management of COVID-19 patients [21-23]. However, studies investigating the impact of anthelmintic treatments on pathogens other than the targeted helminths remain rare. Moreso, studies on effects of albendazole anthelminthic drug on cytokine level of healthy individual are scarce. The authors of the present study hypothesized that since helminth are known to stimulate inflammatory response in the hosts as done by SARS-COV 2, it is likely that albendazole anthelminthic drug which reverses systemic inflammation might reduce cytokine storm in COVID-19 patients. We therefore assessed the levels of three cytokines (IFN- $\gamma$, IL-4 and IL-8) implicated in the severity and pathogenesis of SARS-COV 2.

\section{MATERIALS AND METHODS}

A total of 20 apparently healthy and consented adult (aged between 18-35 years) participants were recruited from staff of University College Hospital, Ibadan, Nigeria and those with the following conditions were excluded:

- Participants that gave no consent or withdrew from the study during the period of the study.

- Participants with clinical features of parasitic, bacterial or viral infection.

- Participants with history of heart disease, hypertension, diabetes, smoking and use of compulsory drugs.

Blood sample was collected before and at 4weeks of anthelminthic drug administration.Four hundred milligram (400mg) single dose of albendazole tablet (Glaxosmithkline) was orally administered to each participant. The serum levels of cytokines (IFN- $\gamma$, IL-4 and IL-8) were determined using Enzyme Linked Immunosorbent Assay (ELISA) with kits from manufacturers (Abcam, MA, USA, AssayPro, MO, USA and Calbiotech, USA). The ELISA was performed as previously described [24]. All the reagents, sample and standards were allowed to attain working room temperature prior to commencement of the assay. Stock standard solution was diluted to varying concentration used for the standard curve. $50 \mu$ l of standards and samples were added to each immunoplate well, covered and allowed to stay at room temperature for 2 hours. The immunoplate was washed 5 times using a plate washer (TECAN, Mannedorf, Switzerland). Biotinlyated Human antibody $(50 \mu \mathrm{l})$ to the cytokines and other analytes as applicable was added to each well. It was allowed to stay at room temperature for 2 hours, after which the wash was repeated 5 times. Streptavidin -Preoxidase Conjugate $(50 \mu \mathrm{l})$ was added to each well, incubated for 30 minutes at room temperature and wash was repeated 5 times. Chromogen Substrate $(50 \mu \mathrm{l})$ was added to each well, incubated for 15 minutes inside a dark cupboard to allow the blue colour to develop. Stop Solution $(50 \mu \mathrm{l})$ was added to stop the reaction, changing the colour from blue to yellow. The absorbance of each well was read at $450 \mathrm{~nm}$ with the aid of a microplate reader (SpectraMax 384 Plus (Molecular Devices, USA). Using a four-parameter logistic curve-fit, the unknown sample concentration was extrapolated from the standard curve. Wilcoxon Signed Ranks test was employed to compare cytokines levels. Level of statistical significance was set at $\alpha_{0.05}$. 


\section{RESULTS}

Table 1 compares the serum cytokine median levels of participants before and 4weeks after albendazole administration. Serum median levels of IFN $-\gamma$ and IL- 8 were significantly reduced at 4 weeks post-albendazole administration $(\mathrm{p}<0.05)$ while IL-4 was insignificantly reduced at 4 weeks post-albendazole administration $(\mathrm{p}>0.05)$ compared with median level before albendazole administration.

Table 1: Serum cytokine (IFN- $\gamma$, IL-4 and IL-8) median levels of healthy Nigerian adults before and 4weeks after albendazole treatment

\begin{tabular}{|l|l|l|l|l|}
\hline & Pre-treatment $(\mathrm{n}=20)$ & 4weeks post-treatment $(\mathrm{n}=20)$ & $\mathrm{Z}$ & $\mathrm{p}$-values \\
\hline $\mathrm{IFN}-\gamma(\mathrm{pg} / \mathrm{ml})$ & $103.69(69.00-110.08)$ & $72.80(43.90-96.88)$ & 7.34 & $0.030^{*}$ \\
\hline $\mathrm{IL}-4(\mathrm{pg} / \mathrm{ml})$ & $188.9(99.7-221.3)$ & $119.98(43.6-160.6)$ & 4.78 & 0.080 \\
\hline $\mathrm{IL}-8(\mathrm{pg} / \mathrm{ml})$ & $288.1(177.0-566.2)$ & $166.22(12.89-800.00)$ & 6.77 & $0.041^{*}$ \\
\hline
\end{tabular}

$*$ Significant at $\mathrm{p} \leq 0.05$

${ }^{\mathrm{Z}}$ Significant compared with pre-treatment (Wilcoxon Signed Ranks Test)

\section{DISCUSSION}

COVID-19, a novel Coronavirus disease with high mortality has no existing treatment. Thus, development of effective prevention and treatment is an urgent need, especially for the life-threatening severe cases. Many COVID-19 patients develop acute respiratory distress syndrome (ARDS), which leads to pulmonary edema and lung failure with liver-, heart-, and kidney- damages. These symptoms are associated with a cytokine storm, manifesting elevated serum levels of IL-1 $\beta$, IL-2, IL-7, IL-8, IL-9, IL-10, IL-17, GCSF, GM-CSF, IFN- $\gamma$ and TNF- $\alpha$ [25]. As a result of no proven effective vaccines or therapeutic agents against SAR-COV 2 [1-3], the current clinical management of COVID-19 patients includes infection prevention and control measures or supportive care including supplemental oxygen and mechanical ventilatory support. Our present study proposed albenzole as supportive options with potential that may reduce cytokine storm to repurpose management of COVID-19, which suggests opportunities for further research.

Albendazole has been used extensively for the treatment of a wide range of helminth parasites including hookworms, Ascaris lumbricoides, Trichuris trichiura, Echinococcus sp. and Taenia sp. with few side effects. Albendazole has lower toxicity and lack of significant effects on the intestinal microflora [14-18]. However, studies investigating the impact of anthelmintic treatments on pathogens other than the targeted helminths remain few. Moreso, studies on effects of albendazole anthelminthic drug on cytokine level of healthy individual are scarce. However, treatment with combination of appropriate drugs and albendazole in helminth co-infection with Mycobacterium tuberculosis, HIV-1 and cancers were reported [17-20].

IL-1 $\beta$, IL-6 and TNF- $\alpha$ cause systemic inflammatory symptoms, including fever, while chemokine (IL-8) attracts and recruits more immune infiltrates [25]. IL-8 is a pro-inflammatory cytokine, secreted by different cells including monocytes, neutrophils, T-lymphocytes, dermal fibroblasts, vascular endothelial cells, hepatocytes and keratinocytes [26]. Its major function is neutrophil activation and recruitment [27]. Expression of IL-8, which is an attractant for neutrophils, is also upregulated in the serum of patients with SARS [28]. Consistent with a role for CXCL8 in pathogenesis, severe disease is associated with an increase in the number of neutrophils in the blood [29]. High serum cytokine and chemokine levels in MERS patients correlated with increased neutrophil and monocyte numbers in lungs and in the peripheral blood, suggesting a possible role for these cells in lung pathology [30]. The significantly reduced IL-8 level at one month after anthelminthic drug administration suggests reduced proliferation of neutrophils and eosinophils within one month after anthelminthic drug treatment.

IFN- $\gamma$, is a critical cytokine for adaptive and innate immunity against viral and protozoan infections. It is produced majorly as part of the innate immune response by natural killer cells and by CD4 Th1 and CD8 cytotoxic T-lymphocyte effector $\mathrm{T}$ cells in cases of specific immunity [37]. Studies have demonstrated high serum levels of pro-inflammatory cytokines (IFN$\gamma$ among others) were found in SARS patients with severe disease compared to individuals with uncomplicated SARS [31]. In addition to proinflammatory cytokines and chemokines, individuals with lethal SARS showed elevated levels of IFN (IFN- $\alpha$ and IFN- $\gamma$ ) and IFN-stimulated genes (ISGs) (CXCL10 and CCL-2) compared to healthy controls or individuals with mild-moderate disease [32]. These results [31.32] suggest a possible role for IFNs and ISGs in the immune-pathogenesis of SARS in humans. Thus, dysregulated and/or exaggerated cytokine and chemokine responses by SARS-CoV-infected dendritic cells and macrophages could play an important role in SARS pathogenesis [33]. However, macrophages are functionally diverse and comprise various subtypes, including classically IFN- $\gamma$ activated pro-inflammatory M1 and alternatively activated anti-inflammatory M2 populations. M1 macrophages secrete various proinflammatory mediators, IL- $1 \beta$, TNF, and nitric oxide [37]. In contrast, M2 macrophages release low amounts of pro-inflammatory molecules but higher levels of anti-inflammatory mediators, such as IL-10. It was 
concluded by Huang et al., [34] that an interferongamma-related cytokine storm was induced post SARS coronavirus infection, and this cytokine storm might be involved in the immune-pathological damage in SARS patients. Therefore, reduced IFN- $\gamma$ as a result of albendazole use might be advantageous for COVID-19 patients. Baricitinib, fedratinib, and ruxolitinib are potent and selective JAK-STAT signalling inhibitors approved for indications such as rheumatoid arthritis and myelofibrosis were reported likely to be effective against the consequences of the elevated levels of cytokines (including IFN- $\gamma$ ) typically observed in people with COVID-19 [34]. Exercise, reduced alcohol intake and high intake ofomega-3 fatty acids (include fatty fish, shellfish, and fortified dairy products) were also shown to lower IFN- $\gamma$ levels [39].

IL-4 is an anti-inflammatory cytokine acting as a pleiotropic regulator of numerous immune and inflammatory processes typically secreted by $\mathrm{T}$ helper 2 lymphocytes, mast cells, eosinophils, and basophils and the beneficial actions of IL- 4 are considered to result from the inhibition of the production and release of proinflammatory cytokines, chemokines, proteases, and reactive oxygen species [36]. In COVID-19 patients, IL-4 was increased especially in males compared with females. Also, a networking clusters of cytokines and growth factors, including previously unknown roles of vascular and stromal remodeling, activation of the innate immunity, as well activation of type 2 immune responses were revealed to be involved in the immunopathogenesis of COVID-19 [38]. IL-4 reducing ability of albendazole might be relevant in controlling cytokine storm of COVID-19 patients.

\section{CONCLUSION}

Previous studies showed that IL 4 is important for homeostasis in the immune response and that IFN- $\gamma$ and IL-8 are key mediators of cytokine storm or cytokine release syndrome in COVID-19 patients. Therefore, our observation that albendazole reduces serum IFN- $\gamma$ and IL- 8 in healthy adults make it an hypothetical candidate that might decrease cytokine storm in COVID-19 patients.

Conflicts of Interest: The authors declare that they have no conflicts of interest.

Acknowledgments: We thank the participants for their cooperations throughout the duration of the study.

\section{REFERENCES}

1. Hossen, M. S., Barek, M. A., Jahan, N., \& Islam, M. S. (2020). A Review on Current Repurposing Drugs for the Treatment of COVID-19: Reality and Challenges. SN Comprehensive Clinical Medicine, 1-13.

2. Santos, J., Brierley, S., Gandhi, M. J., Cohen, M. A., Moschella, P. C., \& Declan, A. B. (2020). Repurposing therapeutics for potential treatment of SARS-CoV-2: A review. Viruses, 12(7), 705.
3. Wu, R., Wang, L., Kuo, H. C. D., Shannar, A., Peter, R., Chou, P. J., ... \&Poiani, G. J. (2020). An update on current therapeutic drugs treating COVID-19. Current Pharmacology Reports, 11(1):1-15.

4. Nelemans, T., \&Kikkert, M. (2019). Viral innate immune evasion and the pathogenesis of emerging RNA virus infections. Viruses, 11(10), 961.

5. Bunte, K., \&Beikler, T. (2019). Th17 cells and the IL-23/IL-17 axis in the pathogenesis of periodontitis and immune-mediated inflammatory diseases. International Journal of Molecular Sciences, 20(14), 3394.

6. Huang, C., Wang, Y., Li, X., Ren, L., Zhao, J., $\mathrm{Hu}, \mathrm{Y}$., ...\& Cheng, Z. (2020). Clinical features of patients infected with 2019 novel coronavirus in Wuhan, China. The Lancet, 395(10223), 497-506.

7. Chen, N., Zhou, M., Dong, X., Qu, J., Gong, F., Han, Y., ...\& Yu, T. (2020). Epidemiological and clinical characteristics of 99 cases of 2019 novel coronavirus pneumonia in Wuhan, China: a descriptive study. The Lancet, 395(10223), 507513.

8. Qin, C., Zhou, L., Hu, Z., Zhang, S., Yang, S., Tao, Y., ...\& Tian, D. S. (2020). Dysregulation of immune response in patients with COVID-19 in Wuhan, China. Clinical Infectious Diseases. 71(15):762-768.

9. Liu, J., Li, S., Liu, J., Liang, B., Wang, X., Wang, H., ...\& Xiong, L. (2020). Longitudinal characteristics of lymphocyte responses and cytokine profiles in the peripheral blood of SARSCoV-2 infected patients. EBioMedicine, 102763.

10. Wan, S., Yi, Q., Fan, S., Lv, J., Zhang, X., Guo, L., ...\&Qiang, M. (2020). Characteristics of lymphocyte subsets and cytokines in peripheral blood of 123 hospitalized patients with 2019 novel coronavirus pneumonia (NCP). MedRxiv.

11. Diao, B., Wang, C., Tan, Y., Chen, X., Liu, Y., Ning, L., ...\& Yuan, Z. (2020). Reduction and functional exhaustion of $\mathrm{T}$ cells in patients with coronavirus disease 2019 (COVID-19). Frontiers in Immunology, 11, 827.

12. Yang, L., Gou, J., Gao, J., Huang, L., Zhu, Z., Lan, C., ...\& Liu, H. (2020). Immune Characteristics Predict Outcome of Severe and Critical COVID-19 Patients.

13. Channappanavar, R.,\& Perlman, S. (2017). Pathogenic human coronavirus infections: causes and consequences of cytokine storm and immunopathology. Seminars in Immunopathology. 39: 539-546.

14. Horton, J. (2000).Albendazole: a review of anthelminthic efficacy and safety in humans. Parasitology. 121:S113-132.

15. Lacey, E. (1990). Mode of action of benzimidazoles. Parasitol Today.6: 112-115.

16. Pourgholami, M. H., Woon, L., Almajd, R., Akhter, J., Bowery, P., \& Morris, D. L. (2001). In vitro and in vivo suppression of growth of 
hepatocellular carcinoma cells by albendazole. Cancer Letters, 165(1), 43-49.

17. Morris, D. L., Jourdan, J. L., \&Pourgholami, M. H. (2001). Pilot study of albendazole in patients with advanced malignancy. Oncology, 61(1), 4246.

18. Walson, J. L., Otieno, P. A., Mbuchi, M., Richardson, B. A., Lohman-Payne, B., Macharia, S. W., ...\& John-Stewart, G. C. (2008). Albendazole treatment of HIV-1 and helminth coinfection: a randomized, double blind, placebocontrolled trial. AIDS (London, England), 22(13), 1601-1609.

19. Anuradha, R., Munisankar, S., Bhootra, Y., Dolla, C., Kumaran, P., Nutman, T. B., \&Babu, S. (2017). Anthelmintic therapy modifies the systemic and mycobacterial antigen-stimulated cytokine profile in helminth-latent Mycobacterium tuberculosis coinfection. Infection and immunity, 85(4).

20. Arinola O. G., \&Edem, V. F. (2020).Levels of Interleukin-8 and Catalase Have Correlations with Zinc in Healthy Adults: Implications for Inflammatory Conditions. Annals of Medical Research. In Press.

21. Jiménez-Pavón, D., Carbonell-Baeza, A., \&Lavie, C. J. (2020). Physical exercise as therapy to fight against the mental and physical consequences of COVID-19 quarantine: Special focus in older people. Progress in Cardiovascular Diseases. 63(3):386-388.

22. Zhang, L., \& Liu, Y. (2020). Potential interventions for novel coronavirus in China: A systematic review. Journal of Medical Virology, 92(5), 479-490.

23. Onifade, A. A., Rafiu, A. T., \&Arinola, O. G. (2020). Serum Chemokine Levels in HIV-infected Nigerian Patients. Archives of Basic and Applied Medicine, 8(1), 17-19.

24. Huang, C., Wang, Y., Li, X., Ren, L., Zhao, J., $\mathrm{Hu}, \mathrm{Y} .$, ...\& Cheng, Z. (2020). Clinical features of patients infected with 2019 novel coronavirus in Wuhan, China. The Lancet, 395(10223), 497-506.

25. Ghasemi, H., Ghazanfari, T., Yaraee, R., Faghihzadeh, S., \& Hassan, Z. M. (2011). Roles of IL-8 in ocular inflammations: a review. Ocular Immunology and Inflammation, 19(6), 401-412.

26. Alfaro, C., Sanmamed, M. F., Rodríguez-Ruiz, M. E., Teijeira, Á.,Oñate, C., González, Á., ... \&Melero, I. (2017). Interleukin-8 in cancer pathogenesis, treatment and follow-up. Cancer Treatment Reviews, 60, 24-31.

27. Wang, W. K., Chen, S. Y., Liu, I. J., Kao, C. L., Chen, H. L., Chiang, B. L., ... \& Yang, P. C. (2004). Temporal Relationship of Viral Load, Ribavirin, Interleukin (IL)-6, IL-8, and Clinical Progression in Patients with Severe Acute Respiratory Syndrome. Clinical Infectious Diseases, 39(7), 1071-1075.
28. Lo, I. L., Lio, C. F., Cheong, H. H., Lei, C. I., Cheong, T. H., Zhong, X., ... \& Sin, N. N. (2020). Evaluation of SARS-CoV-2 RNA shedding in clinical specimens and clinical characteristics of 10 patients with COVID-19 in Macau. International Journal of Biological Sciences, 16(10), 1698.

29. Kim, E. S., Choe, P. G., Park, W. B., Oh, H. S., Kim, E. J., Nam, E. Y., ...\& Park, S. W. (2016). Clinical progression and cytokine profiles of Middle East respiratory syndrome coronavirus infection. Journal of Korean Medical Science, 31(11), 1717-1725.

30. Cameron, M. J., Bermejo-Martin, J. F., Danesh, A., Muller, M. P., \& Kelvin, D. J. (2008). Human immunopathogenesis of severe acute respiratory syndrome (SARS). Virus Research, 133(1), 13-19.

31. Cameron, M. J., Ran, L., Xu, L., Danesh, A., Bermejo-Martin, J. F., Cameron, C. M., ...\& Willey, B. M. (2007). Interferon-mediated immunopathological events are associated with atypical innate and adaptive immune responses in patients with severe acute respiratory syndrome. Journal of Virology,81(16), 86928706.

32. Chien, J. Y., Hsueh, P. R., Cheng, W. C., Yu, C. J., \& Yang, P. C. (2006). Temporal changes in cytokine/chemokine profiles and pulmonary involvement in severe acute respiratory syndrome. Respirology, 11(6), 715-722.

33. Huang, K. J., Su, I. J., Theron, M., Wu, Y. C., Lai, S. K., Liu, C. C., \& Lei, H. Y. (2005). An interferon $\gamma$ related cytokine storm in SARS patients. Journal of Medical Virology, 75(2), 185194.

34. Huang, C., Wang, Y., Li, X., Ren, L., Zhao, J., $\mathrm{Hu}$, Y., ...\& Cheng, Z. (2020). Clinical features of patients infected with 2019 novel coronavirus in Wuhan, China. The Lancet, 395(10223), 497-506.

35. Wills-Karp, M., \&Finkelman, F. D. (2008). Untangling the complex web of IL-4-and IL-13mediated signaling pathways. Science Signaling, 1(51), pe55.

36. Kak, G., Raza, M., \&Tiwari, B. K. (2018). Interferon-gamma (IFN- $\gamma)$ : exploring its implications in infectious diseases. Biomolecular Concepts, 9(1), 64-79.

37. Petrey, A. C., Qeadan, F., Middleton, E. A., Pinchuk, I. V., Campbell, R. A., \&Beswick, E. J. (2020). Cytokine release syndrome in COVID 19: Innate immune, vascular, and platelet pathogenic factors differ in severity of disease and sex. Journal of Leukocyte Biology.

38. Feng, C., Keisler, D. H., \&Fritsche, K. L. (1999). Dietary omega-3 polyunsaturated fatty acids reduce IFN-gamma receptor expression in mice. Journal of Interferon \& Cytokine Research, 19(1), 41-48. 